\title{
Cystadenoma and cystadenocarcinoma of the pancreas
}

\author{
J. A. CAMPBELL AND A. H. CRUICKSHANK \\ From the Departments of Pathology, Broadgreen Hospital, Liverpool, \\ and the University of Liverpool
}

SYNOPSIS The pathological findings in 14 cases of cystadenoma and three cases of cystadenocarcinoma of the pancreas have been studied in relation to the clinical effects. All the patients were women, and their ages ranged from 13 to 90 years. Four tumours measuring 2 to $4 \mathrm{~cm}$. in diameteid had not caused symptoms or signs. Tumours from 7 to $12 \mathrm{~cm}$. in diameter had been felt in the upper abdomen and half the patients with such tumours had complained of pain. In two cases with large tumours there was severe bleeding into the alimentary canal.

The cystadenomas were of two types: 1 Tumours with small loculi lined by cubical epitheliume in which there was little secretion of mucus by the epithelium but abundant mucoid stroma. There were six of these tumours and we have no evidence of malignant change in this type. 2 Tumours with relatively large cystic spaces filled with mucus and lined by tall, mucus-secreting epithelium?. There were eight of these, and the possibility of malignant transformation in this type of tumoug indicated by the finding that in three cases of cystadenocarcinoma two appeared to have beent preceded by simple mucus-secreting cystadenomas.

Signs of recent or old haemorrhage were common in both types of cystadenoma and also in cystadenocarcinomas.

Previous studies of cystadenoma and cystadenocarcinoma of the pancreas have been on quite small numbers of cases. Thus Bowers, Lord, and McSwain (1942) reported five cases of cystadenoma and Mahorner and Mattson (1931) four of cystadenocarcinoma, while many reports have been concerned with even smaller numbers of cases. Some authors have supplemented their own observations by an analysis of accounts published by others, e.g., Zintel, Enterline, and Rhoads (1954) in the case of cystadenoma, and Cornes and Azzopardi (1959) in the case of cystadenocarcinoma, to quote some fairly recent examples. We feel that our collection of 14 cases of cystadenoma and three cases of cystadenocarcinoma, although small, allows us to offer some original observations about these tumours. In particular, we wish to draw attention to two distinct types of cystadenoma of the pancreas, each with its own gross and microscopic appearances.

\section{SEX AND AGE}

All the tumours, both simple and malignant, occurred in women. The patients' ages ranged from 13 to years. At least one case occurred in each decade between these limits and four patients with cysta denoma and one with cystadenocarcinoma were their seventies.

\section{SIZE IN RELATION TO CLINICAL EFFECTS}

All three cystadenocarcinomas caused clinical effect? The following table is presented to correlate the size and site of the cystadenomas with the presence or absence of clinical effects.

TABLE

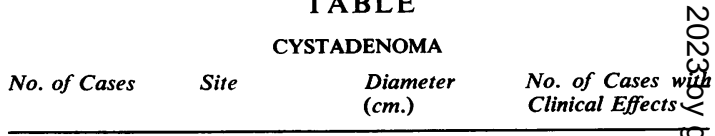

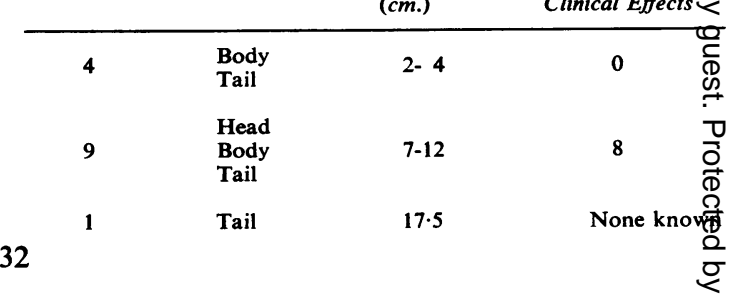




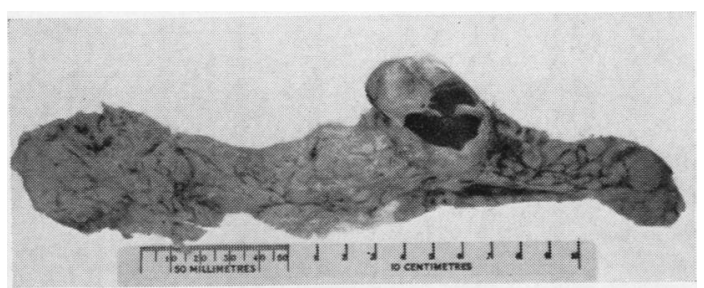

FIG. 1

FIG. 1. Mucus-secreting cystadenoma about $4 \mathrm{~cm}$. in diameter at the junction of the body and tail of the pancreas, found at necropsy in a 79-year-old woman who had died suddenly of coronary occlusion.

FIG. 2. A small-locular cystadenoma, $7 \mathrm{~cm}$. in diameter, from the front of the head of the pancreas in a woman of 78 . The cystic spaces are embedded in myxomatous stroma.

The four tumours measuring $4 \mathrm{~cm}$. or less in diameter, all in the body or tail of the pancreas, had been symptomless and undetected during life, and, when found at necropsy, did not appear to have contributed in any way to the cause of death. Fig. 1 is a photograph of one of the tumours within this range.

Of the tumours between 7 and $12 \mathrm{~cm}$. in diameter, eight out of nine had been detected either by the patient herself or by her doctor as an upper abdominal mass, and four of these had also caused pain, usually intermittent and colicky. Two of the tumours in this range also caused severe bleeding into the alimentary canal. The largest tumour of all may have been detected by the patient herself but she did not consult her doctor about it and seemed to her friends to be in good health up to the time when she died of accidental carbon monoxide poisoning. The tumour was discovered during the postmortem examination ordered by the Coroner. None of the cases of cystadenoma or of cystadenocarcinoma had diabetes.

\section{APPEARANCES}

The cystadenomas conformed to two types, each of which had its characteristic macroscopic and microscopic appearances. Intermediate forms were not encountered.

The naked-eye appearances of the first are shown in Fig. 2. The cystic loculi were small, less than $1 \mathrm{~cm}$. across, and were embedded in an abundant myxomatous stroma which was most obvious in the centre of the cut surface and became less conspicuous peripherally giving an irregularly radiating appearance.

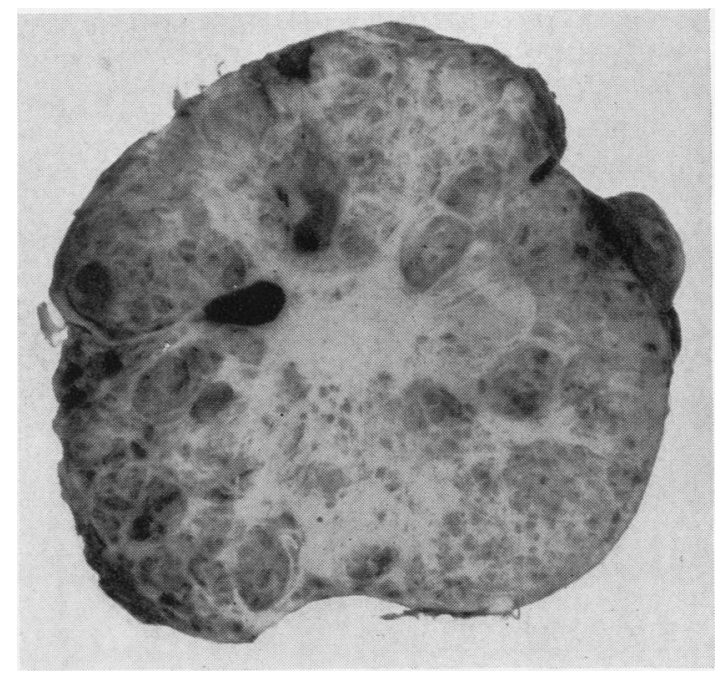

FIG. 2

Microscopically the cystic loculi varied in size from minute spaces to those seen with the naked eye. They were lined by low cubical epithelium (Fig. 3) with little obvious mucus, either in the epithelial cells or in the fluid that filled the cavities. Mucoid material was abundant, however, in the stroma (Fig. 4) and some of the very smallest acinar spaces contained material that stained as mucus in alcian blue and mucicarmine preparations. Two of the smaller tumours had microscopic foci of calcification in the stroma, and in one of the tumours microscopic papillary epithelial projections were fairly marked. There was nothing in the six cystadenomas with these characteristics to suggest malignant transformation in any of them. They were relatively small, their diameters ranging from 2 to $8 \mathrm{~cm}$., with an average diameter of $5.17 \mathrm{~cm}$.

The second type of cystadenoma is shown macroscopically in Fig. 5. The cystic loculi were much larger and were filled with thick viscid mucus. They varied in size from about $1 \mathrm{~cm}$. in diameter to 5 or $6 \mathrm{~cm}$. Microscopically the spaces were lined by tall, mucus-secreting cells (Fig. 6). Papillary epithelial projections were marked in the smaller loculi. No mucoid appearance was present in the relatively scanty fibrous stroma between the loculi. The vacuoles in the epithelial cells stained vividly with mucicarmine and alcian blue; the stroma did not take up these stains.

There were eight cystadenomas of this type and they tended to be larger than the preceding type, ranging from 4 to $17.5 \mathrm{~cm}$. in diameter, with an average diameter of $11 \mathrm{~cm}$. The cases of cystadenocarcinoma to be described later appeared to be closely related to this type of cystadenoma. 


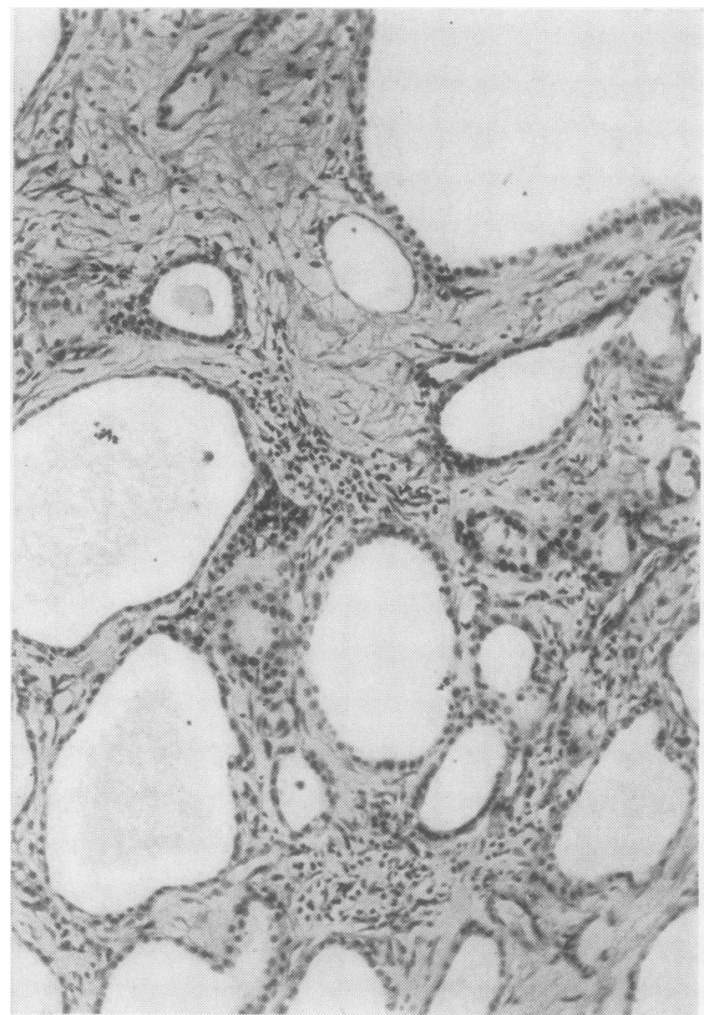

FIG. 3

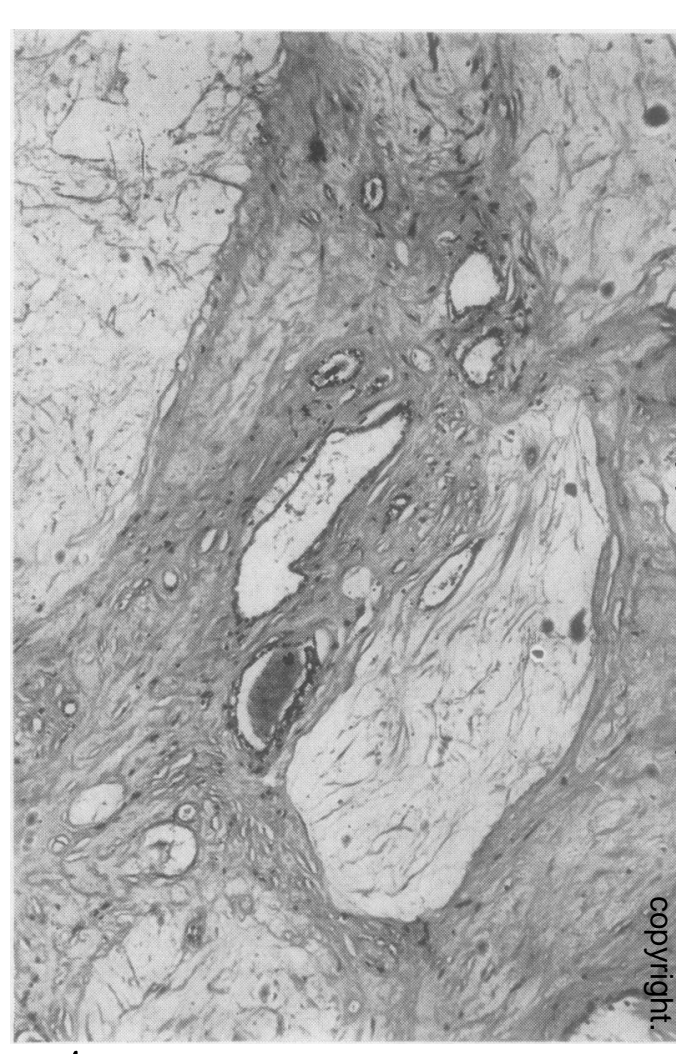

FIG. 4

FIG. 3. Small-locular type of cystadenoma in which the spaces are lined by low epithelium that is not secreting mucu§ Haematoxylin and eosin $\times 120$.

FIG. 4. Myxomatous stroma of the small-locular type of cystadenoma. Haematoxylin and eosin $\times 60$.

\section{HAEMORRHAGE}

Recently extravasated red cells and haemosiderinfilled macrophages were common in both types of tumour and sometimes there was gross haemorrhage throughout the tumour or into a single loculus. In one of the two cases with alimentary bleeding the tumour was found at operation to be compressing the vasa brevia draining from the stomach to the splenic vein with consequent hyperaemia of the gastric mucosa. After the tumour had been removed there was no recurrence of the previously frequent episodes of haematemesis and melaena. The clinical aspects of the case have been described by Grunberg, Blair, and St. Hill (1952).

In the other case, an episode of haematemesis was followed by decrease in the size of the abdominal swelling and at operation the cyst was so firmly adherent to the posterior wall of the stomach that part of the stomach had to be removed with the cyst.
The alimentary bleeding in this case was thought to have been due to rupture of a haemorrhagic loculus of the tumour into the stomach, but the tumour had. to be removed in several pieces and the rupture coulg not be identified when the specimen reached the laboratory. The patient has been in good healtb since the operation. The microscopic appearance of a section of the tumour is shown in Fig. 6.

\section{MALIGNANT TRANSFORMATION}

The following case is believed to be an example of cystadenoma with superimposed malignancy.

A 48-year-old married woman, who had vomited bloog on two occasions during the preceding three months, was operated upon in June 1949. The gall bladder containe stones and was removed. A cyst with two main loculi was seen in the pancreas. One loculus contained mucus, the other old blood. Complete removal of the cyst was nof possible and the part that could not be excised w\$ 


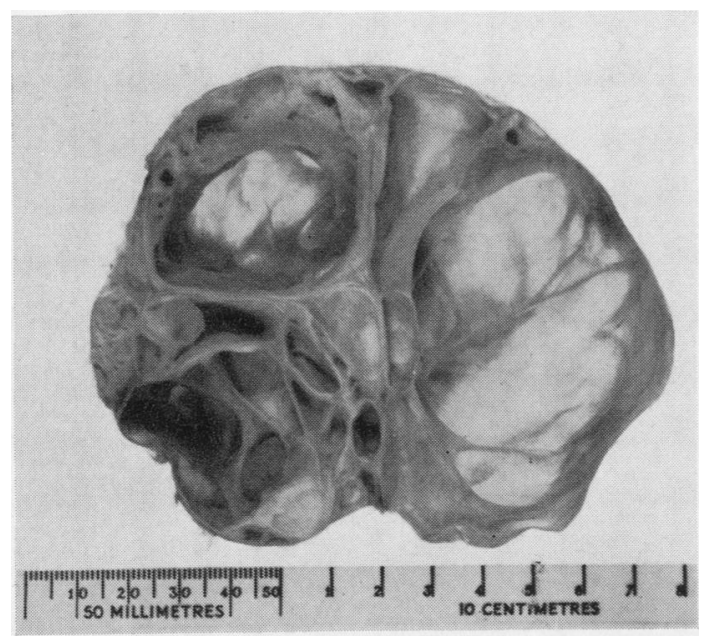

FIG. 5. Loculi from which mucus has been removed in a large-locular type of cystadenoma removed surgically from a girl of 13 .

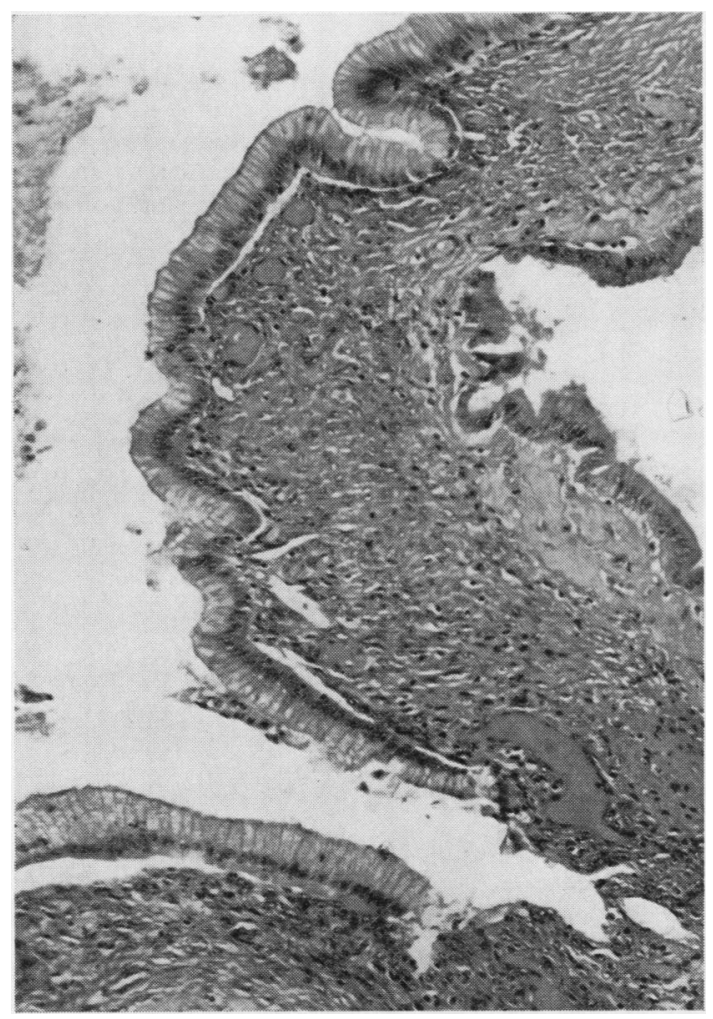

FIG. 6. Mucus-secreting epithelium lining the loculi of a large-locular type of cystadenoma removed surgically from $a$ woman of 37. Haematoxylin and eosin $\times 120$. marsupialized by being stitched to the abdominal wall. The fistula which followed was very troublesome but, in 1953, following a course of radiotherapy, the fistula closed and all discharge ceased. In February 1961, however, the fistula became active again and malignant nodules appeared in the skin at its margin. The skin nodules, the fistula, a cystic tumour in the region of the tail of the pancreas, and the spleen were excised. The gross appearance of the cystic tumour is shown in Fig. 7. Microscopically all transitions between well-differentiated mucus-secreting cystadenoma, cystadenocarcinoma, and undifferentiated carcinoma could be recognized (Figs. 8 and 9). These appearances, combined with the history of sudden reactivation of a tumour that had seemed to have been dormant for about six years are taken to indicate that the tumour, after behaving as a cystadenoma for some years, became carcinomatous.

Following the excision, the tumour recurred in the abdominal wall. Death was from pulmonary embolism and, at necropsy, secondary carcinoma was found in lymph nodes in the upper abdomen.

The second case of cystadenocarcinoma was an unmarried woman of 73 who was admitted to hospital because of an upper abdominal mass. An abscess, which formed part of the mass, was drained. A week later pulmonary embolism caused death and, at necropsy, a cystadenocarcinoma of the tail of the pancreas was found to be invading the spleen and to have caused small fistulae into both the stomach and the splenic flexure of the colon. The tumour did not involve the adjacent lymph nodes or any distant organs, its malignant behaviour being limited to local invasiveness. The contents of the cavity of the tumour and the walls of the tumour were so rich in haemosiderin that haemorrhage into the alimentary canal through the fistulae must have taken place from time to time, although no clinical evidence of such bleeding was recorded in the case notes.

The third case of cystadenocarcinoma was a married woman of 47 who had an upper abdominal mass combined with many deposits of secondary tumour when she was first admitted to hospital. Very poorly differentiated carcinoma, whose structure gave no clue to the site of the primary tumour, was found on biopsy of a superficial nodule. Death occurred very quickly and, at necropsy, the tumour shown in Fig. 10 was found in the pancreas. Microscopically the tumour contained well-differentiated cystadenomatous areas, secreting mucus, along with areas of very poorly differentiated carcinoma. The two types of structure were taken to indicate carcinomatous change in a pre-existing cystadenoma.

\section{DISCUSSION}

The absence of males among the cases is a clear demonstration of the tendency of these tumours to affect women more commonly than men, as has been pointed out by several of the authors who have reviewed the published cases, e.g., Benson and Gordon (1947) who found a sex ratio of eight females to one male and Mozan (1951) who found a very similar ratio of seven females to one male. 


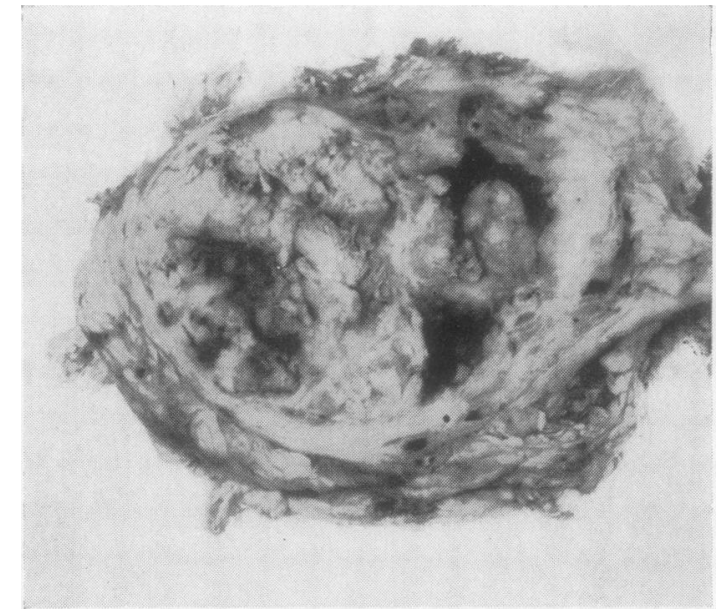

FIG. 7. A cystadenocarcinoma, $9 \mathrm{~cm}$. in diameter, removed surgically from a woman of 59 years of age.

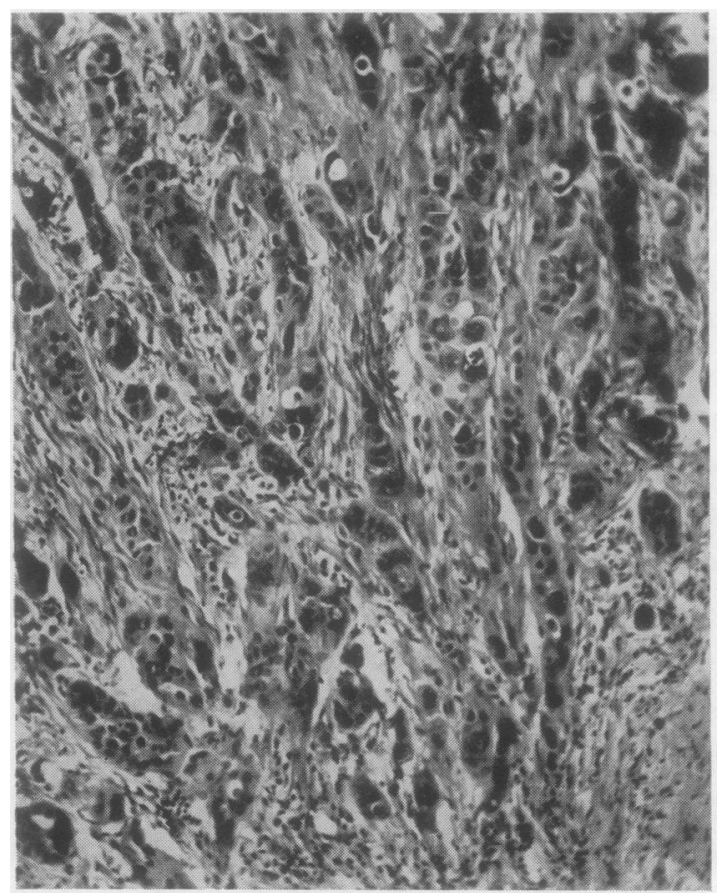

FIG. 8. Well-differentiated and less well-differentiated areas in a cystadenocarcinoma. Haematoxylin and eosin $\times 32$.
FIG. 9. Poorly differentiated area of the same cystadeño carcinoma as that shown in Fig. 8. Haematoxylin and eosin $\times 120$.

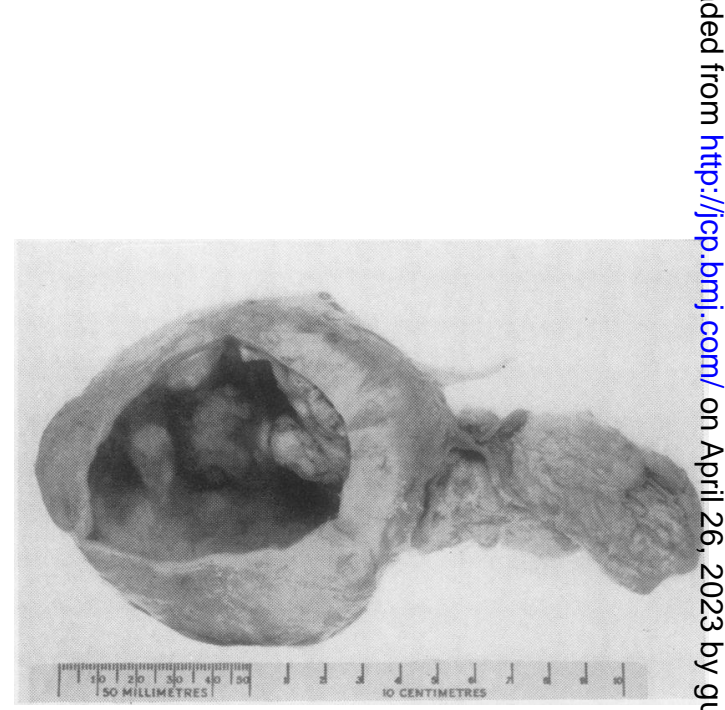

FIG. 10. A cystadenocarcinoma in the tail of the pancreas has had an opening made in its posterior wall. 
This has not been the experience of all however, e.g., Carling Rock, and Hicks (1925) and Smith (1958).

The absence of diabetes is not what might be expected in the light of previous articles and reviews, e.g., six out of 28 cases were diabetic in the series of Benson and Gordon (1947) and three out of five in that of Bowers et al. (1942). Only one of the cases in the present series had transient hyperglycaemia while recovering from the excision of the tumour. Another, a woman, 78 years old when the tumour was excised, developed mild diabetes mellitus two years after the operation: this is regarded as coincidence. The lack of post-operative diabetes is all the more surprising in that the non-neoplastic pancreatic tissue excised with several of the tumours appeared to be unusually rich in islets, probably because of pressure atrophy of the externally-secreting pancreatic tissue.

No patient had jaundice and, in the three cases in which the tumour was in the head of the pancreas, the common bile duct was not compressed.

The episodes of alimentary bleeding seem to be a well-recognized complication. Positive tests for occult blood in the faeces have been mentioned by several authors, and Malcolm (1906) described the case of a woman who had a sudden alimentary haemorrhage that was so severe that she fainted.

Estimations of the enzymes present in the fluid in the cystic parts of the tumours were possible in only two cases. A small amount of amylase was found in one; neither trypsin nor amylase could be detected in the other. Both these tumours were of the mucussecreting type. These findings are in keeping with the commonly held opinion that pancreatic cystadenomas are tumours of the epithelium of the pancreatic ducts rather than of pancreatic glandular tissue: an absence of pancreatic enzymes is the rule (Smith, 1958).

Eventual malignant behaviour after a long period of enlargement without invasion, as in the first case of cystadenocarcinoma described in this paper, has been described by others who have studied cystic pancreatic tumours (Lichtenstein, 1934; Smith, 1958). The tendency for invasiveness to be local at first was a feature of the case reported by Speese (1915) which was remarkably similar to one of the cases reported here. The case of Young (1937) also passed through a phase of local invasion of the abdominal wall before final dissemination to many organs took place.

The insignificant amount of calcification in our cases both of cystadenoma and of cystadenocarcinoma is not in accord with the experience of Herrman and Asbell (1949), Haukohl and Melamed (1950), Smith (1958), and Cornes and Azzopardi (1959), to quote some who have commented on this feature. Sections from all our cases were stained by von Kossa's method but it was not possible to examine all our gross specimens radiologically. The three cystadenocarcinomas, however, were all examined along with a number of the cystadenomas. In only one of the tumours, the cystadenocarcinoma that contained much haemosiderin, was a small area of calcification that had not been observed with the naked eye or in sections seen in the radiograph. Calcification was not a conspicuous feature in any of our cases.

We are indebted to Professor Charles Wells for allowing us to use specimens in the Department of Surgery of the University of Liverpool and to Dr. C. A. St. Hill of the Royal Southern Hospital, Liverpool, and to Dr. Freda Roberts of the Stanley Hospital, Liverpool, for having lent specimens.

The photography is the work of Mr. F. Beckwith, F.I.M.L.T., of the Department of Pathology, University of Liverpool.

\section{REFERENCES}

Benson, R. E., and Gordon, W. (1947). Surgery, 21, 353.

Bowers, R. F., Lord, J. W., and McSwain, B. (1942). Arch. Surg., 45, 111.

Carling, E. Rock, and Hicks, J. A. B. (1925). Brit. J. Surg., 13, 238.

Cornes, J. S., and Azzopardi, J. G. (1959). Ibid., 47, 139.

Grunberg, A., Blair, J. L., and St. Hill, C. A. (1952). Brit. med. J., 2, 265.

Haukohl, R. S., and Melamed, A. (1950). Amer. J. Roentgenol., 63, 234.

Herrman, C. S., and Asbell, N. (1949). Amer. J. Surg., 78, 107.

Lichtenstein, L. (1934). Amer. J. Cancer., 21, 542.

Mahorner, H. R., and Mattson, H. (1931). Arch. Surg., 22, 1018.

Malcolm, J. D. (1906). Lancet, 1, 1676.

Mozan, A. A. (1951). Amer. J. Surg., 81, 204.

Smith, R. (1958). In Cancer, ed. R. W. Raven. Vol. 2, p. 186, and p. 198. Butterworth, London.

Speese, J. J. (1915). Ann. Surg., 61, 759.

Young, E. L. (1937). New Engl. J. Med., 216, 334.

Zintel, H. A., Enterline, H. T., and Rhoads, J. E. (1954). Surgery, 35, 612. 